ORIGINAL ARTICLE

\title{
Impact of positive legionella urinary antigen test on patient management and improvement of antibiotic use
}

\author{
J Garbino, J-E Bornand, I Uçkay, S Fonseca, H Sax
}

J Clin Pathol 2004;57:1302-1305. doi: 10.1136/icp.2004.018861

See end of article for authors' affiliations

.....................

Correspondence to: Dr J Garbino, Division of Infectious Diseases, Department of Internal Medicine, University of Geneva Hospitals, 24, rue Micheli-du-Crest, 1211

Geneva 14, Switzerland; jorge.garbino@hcuge.ch

Accepted for publication 12 August 2004

\begin{abstract}
Aim: To assess the incidence of legionella infection over a 27 month period at a large university hospital. Material and Methods: The present retrospective cohort study enrolled patients with legionellosis, defined as those presenting a positive urinary antigen for legionella together with a medical history, clinical findings, and radiological findings consistent with pneumonia. These patients were evaluated to determine the relation between their test results and changes in treatment modalities. A control group of patients with pneumonia but a negative urinary antigen test for legionella were also analysed.

Results: Twenty seven of 792 assessed patients tested positive for legionella. In 22 of these patients, legionella active antibiotics were administered empirically. In seven patients, the test results prompted a legionella specific treatment, whereas in 12 cases, non-specific antibiotics were stopped within 24 hours. Overall, treatment was altered in more than half of the patients as a result of the test results.

Conclusions: The urinary antigen may have a direct impact on clinical management of pulmonary legionellosis. However, patient comorbidities and individual clinical judgment are still important for determining the best treatment to be given in each individual case.
\end{abstract}

egionnaires' disease is an important cause of community and hospital acquired pneumonia. ${ }^{1}$ It figures prominently - among the four most common causes of community acquired pneumonia requiring hospitalisation. ${ }^{2}$ Among these pneumonias, legionellosis is a severe disease with a mortality rate of up to $20 \%{ }^{1}$ The aetiological diagnosis of legionellosis was difficult before the introduction of the urinary antigen test. The clinical symptoms of the disease are not specific for legionellosis and do not contribute to establishing an accurate diagnosis. ${ }^{3}$ Laboratory evaluation requires expertise and involves specific culture media that are only used upon clinical suspicion of legionellosis. ${ }^{4}$ Direct immunofluorescence tests are operator dependent and of low yield. Serological testing does not have an impact on patient management because seroconversion occurs relatively late in the course of infection.

"The aetiological diagnosis of legionellosis was difficult before the introduction of the urinary antigen test"

Legionella is susceptible to various classes of antibiotics, except for betalactams, cephalosporins, and carbapenems, both in vitro and in clinical disease. ${ }^{1}$ Drugs of choice include macrolides-such as erythromycin, clarithromycin, or azithromycin-and fluoroquinolones-such as ciprofloxacin, levofloxacin, or moxifloxacin-with a recommended treatment duration of three weeks. ${ }^{5}$

The urinary antigen test facilitates the diagnosis of a large number of cases of Legionella pneumophila pneumonia, and is therefore extremely useful to clinicians for both diagnosis and therapeutic decision making. We investigated the impact of the urinary antigen test on patient management, with respect to therapeutic decision making on an everyday basis.

\section{METHODS}

\section{Setting and laboratory procedures}

The University of Geneva Hospitals, Switzerland, comprises a tertiary care institution featuring 1200 acute care and 1000 longterm care beds, with 45000 annual admissions. The institution's virology laboratory receives all the hospital's samples for legionella urinary antigen testing.

A commercially available enzyme immunoassay kit (Legionella Urine Antigen EIA ${ }^{\circledR}$; Biotest AG, Dreieich, Germany) was introduced into the clinical setting in 1997. This test is designed to detect $L$ pneumophila serogroup 1 (sensitivity, 94.6\%), but also has some crossreactivity with other serogroups ( $86 \%$ sensitivity for serogroups $2,3,4,6$, and 10), in addition to other legionella species. ${ }^{6}$ The kit is a standard "sandwich" enzyme linked immunosorbent assay, which uses a polyclonal rabbit antiserum to capture soluble antigen and peroxidase labelled rabbit antibodies to detect immobilised antigen. Specificity is reported to be $100 \%$, and sensitivity is reported to be $94.6 \%$ for L pneumophila serogroup 1 and $86 \%$ for legionellosis of any serogroup. ${ }^{6}$ In another study, sensitivity was reported to be $76 \%$ for community acquired cases. ${ }^{7}$

\section{Study design}

This was a retrospective, descriptive, cohort study over a period of 27 months encompassing all adult patients with legionellosis admitted to our hospital and patients with nosocomial legionellosis. A patient with legionellosis was defined as presenting a positive urinary antigen test along with a medical history and clinical and radiological findings consistent with pneumonia (new infiltrates on chest $x$ ray). In addition, at least two of the following findings were included: cough or increasing severity of cough, acute changes in the quality of sputum, fever, auscultatory findings such as rales or evidence of pulmonary consolidation, dyspnoea, and leucocytosis. ${ }^{8}$ According to the Centers for Disease Control and Prevention, a nosocomial case required onset of pneumonia symptoms after 10 days of hospitalisation, and a probable nosocomial case exhibited onset between the second and 10th day of hospitalisation. ${ }^{9}$ The remaining cases were considered to be community acquired. The urinary antigen result was assumed to have an impact on the initiation of legionella active treatment or the stopping of non-active treatment if active treatment was initiated or 
Table 1 Characteristics of the 27 study patients with pneumonia and positive urinary antigen test

\begin{tabular}{|c|c|c|c|c|c|c|c|c|c|c|}
\hline Sex & $\begin{array}{l}\text { Age } \\
\text { (years) }\end{array}$ & $\begin{array}{l}\text { Diagnosis at } \\
\text { admission }\end{array}$ & $\begin{array}{l}\text { Main underlying } \\
\text { diseases }\end{array}$ & $\begin{array}{l}\text { Stay in } \\
\text { ICU/FO }\end{array}$ & $\begin{array}{l}\text { Delay } \\
\text { between A } \\
\text { and } T^{*}\end{array}$ & $\begin{array}{l}\text { Delay } \\
\text { between } T \\
\text { and } R^{*}\end{array}$ & $\begin{array}{l}\text { Delay } \\
\text { between } \mathrm{R} \\
\text { and ST } \dagger^{*}\end{array}$ & $\begin{array}{l}\text { Delay } \\
\text { between R } \\
\text { and NST* }\end{array}$ & $\begin{array}{l}\text { Nosocomial } \\
\text { legionellosis }\end{array}$ & Impacł‡ \\
\hline 1 Female & 60 & $\begin{array}{l}\text { Fulminant } \\
\text { hepatitis }\end{array}$ & Diabetes & Yes/Yes & 11 & 1 & NA & NA & Definite & No/No \\
\hline 2 Male & 50 & Pneumonia & None & Yes/No & 4 & 1 & -5 & 0 & No & No/Yes \\
\hline 3 Male & 59 & Pneumonia & Thrombosis & $\mathrm{No} / \mathrm{No}$ & 2 & 1 & 0 & 2 & No & Yes/No \\
\hline 4 Female & 30 & $\begin{array}{l}\text { Fever/ } \\
\text { neutropenia }\end{array}$ & $\mathrm{HD}$ & No/No & 2 & 0 & 0 & 10 & No & Yes/No \\
\hline 5 Female & 37 & Pneumonia & AIDS, HCV & $\mathrm{No} / \mathrm{No}$ & 1 & 3 & 0 & 4 & No & Yes/No \\
\hline 6 Female & 46 & Pneumonia & Alcoholism & $\mathrm{No} / \mathrm{No}$ & 3 & 1 & -3 & 0 & No & No/Yes \\
\hline 7 Male & 60 & Pneumonia & Stroke, hypertension & $\mathrm{No} / \mathrm{No}$ & 4 & 2 & -5 & 0 & No & $\mathrm{No} / \mathrm{Yes}$ \\
\hline 8 Male & 47 & Pneumonia & None & Yes/No & 2 & 0 & -2 & 1 & No & No/Yes \\
\hline 9 Female & 3 & $\begin{array}{l}\text { Fallot's } \\
\text { tetralogy }\end{array}$ & Fallot's tetralogy, PC & Yes/No & 20 & 2 & -2 & 2 & Definite & No/No \\
\hline 10 Female & 82 & $\begin{array}{l}\text { Myocardial } \\
\text { infarct }\end{array}$ & Heart disease, WG & Yes/No & 12 & 3 & -3 & -3 & Definite & No/No \\
\hline 11 Female & 78 & Pneumonia & Hypertension & Yes/No & 1 & 2 & -2 & 0 & No & No/Yes \\
\hline 12 Female & 43 & Pneumonia & None & $\mathrm{No} / \mathrm{No}$ & 0 & 2 & 0 & 0 & No & Yes/Yes \\
\hline 13 Male & 39 & Pneumonia & Pneumothorax & Yes/No & 1 & 5 & -5 & 2 & No & $\mathrm{No} / \mathrm{No}$ \\
\hline 14 Male & 75 & Pneumonia & $C D$ & Yes/Yes & 37 & 2 & 0 & 12 & Definite & Yes/No \\
\hline 15 Male & 61 & Pneumonia & Hypertension & Yes/No & 1 & 2 & -2 & 1 & No & $\mathrm{No} / \mathrm{Yes}$ \\
\hline 16 Female & 67 & Pneumonia & $\begin{array}{l}\text { Rheumatoid } \\
\text { polyarthritis }\end{array}$ & No/No & 3 & 0 & -1 & 0 & No & No/Yes \\
\hline 17 Female & 63 & Pneumonia & COPD & Yes/No & 0 & 4 & -3 & -4 & No & No/No \\
\hline 18 Male & 39 & Pneumonia & AIDS & No/No & 0 & 4 & -4 & 0 & No & No/Yes \\
\hline 19 Male & 58 & $\begin{array}{l}\text { Renal } \\
\text { Insufficiency }\end{array}$ & $\begin{array}{l}\text { GS, renal } \\
\text { transplantation }\end{array}$ & Yes/No & 18 & 5 & -7 & 5 & $\begin{array}{l}\text { No (infection } \\
\text { during home } \\
\text { leave) }\end{array}$ & $\mathrm{No} / \mathrm{No}$ \\
\hline 20 Male & 78 & Diverticulitis & Asthma & Yes/No & 2 & 1 & -1 & -3 & No & No/No \\
\hline 21 Male & 74 & Pneumonia & Cardiac failure & No/No & 2 & 1 & 0 & -2 & No & Yes/No \\
\hline 22 Male & 55 & Pneumonia & Alcoholism & Yes/No & 2 & 0 & -2 & 3 & No & No/No \\
\hline 23 Male & 51 & Pneumonia & Hypercholesterolaemia & $\mathrm{No} / \mathrm{No}$ & 2 & 2 & -4 & 0 & No & No/Yes \\
\hline 24 Female & 75 & Pneumonia & COPD & Yes/No & 0 & 1 & -1 & 13 & No & $\mathrm{No} / \mathrm{No}$ \\
\hline 25 Female & 63 & Pneumonia & Hypertension & $\mathrm{No} / \mathrm{No}$ & 1 & 2 & 0 & 0 & No & Yes/Yes \\
\hline 26 Male & 79 & Pneumonia & Prostatic carcinoma & Yes/Yes & 1 & 5 & -6 & 2 & No & $\mathrm{No} / \mathrm{No}$ \\
\hline 27 Male & 60 & Pneumonia & Alcoholism & No/No & 0 & 6 & -3 & 0 & No & No/Yes \\
\hline
\end{tabular}

non-active treatment was stopped within one day of the availability of the test result, respectively.

The following study variables were extracted retrospectively from patient records using a standard protocol by an attending infectious diseases physician: patient's age and sex, date of symptom onset, date of hospitalisation, comorbidities, and date of initiation and termination of all antibiotic prescriptions during hospitalisation. Dates of urinary antigen test request and result reporting were extracted from the hospital's laboratory database.

For the same period, we also considered a group of patients as controls. They also had the diagnosis of pneumonia and a legionella urinary antigen test was performed. This control group consisted of 54 patients in whom the urinary antigen was negative, and we examined the impact of the test result on antibiotic treatment.

\section{RESULTS}

\section{Patients}

During the study period, 909 urinary antigen tests for L pneumophila were performed in 792 patients. The 27 (3.4\%) patients who had positive results, 12 females and 15 males, formed the basis of our study. The median age was 60 years (range, 3-82). Eight patients were considered to be immunosuppressed (two AIDS, clinical category B2 and B3; two cancer, Hodgkin disease and colon carcinoma; two autoimmune diseases, Wegener's granulomatosis and Goodpasture's syndrome; one diabetes; one renal transplantation treated with cyclosporine), and six were smokers. All but three patients presented with at least one underlying disease (table 1). In 20 patients, pneumonia was recognised as the main diagnosis at the time of hospital admission. The remaining patients were admitted for myocardial infarction, fulminant hepatitis, fever and neutropenia, Fallot's tetralogy, renal insufficiency, and diverticulitis (table 1). There were four cases of nosocomial legionellosis and three cases were judged as travel associated. The remaining cases were deemed sporadic.

\section{Laboratory results}

Seven of nine patients had an additional positive culture result for $L$ pneumophila serogroup 1 . In six cases, direct fluorescent antibody testing was positive for respiratory secretions. The diagnosis was confirmed in only one patient by all three tests. In 15 patients, the urinary antigen test was the only positive laboratory test. In 13 patients, tracheal secretions were culture positive for legionella (11 bronchoalveolar lavage, two tracheal aspirations). In two patients, Streptococcus pneumoniae and Escherichia faecalis were isolated from bronchoalveolar lavage specimens as additional potential aetiological agents.

The median time from admission to legionella urinary antigen testing was two days (range, $0-37$ ). In 18 patients, the test was ordered within 48 hours of admission, in 22 patients, within four days. Clinicians had to wait a median time of two days (range, 0-6) for the legionella urinary antigen result.

\section{Treatment}

All patients received empirical antibiotic treatment at admission, including a betalactam (16 patients), a cephalosporin 
( six patients), a carbapenem (four patients), or a macrolide antibiotic alone (one patient). Initial treatment included legionella active antibiotics in 22 cases: 19 patients received clarithromycin, and three received erythromycin. Of the five remaining patients, four were given an appropriate treatment only upon receipt of the positive urinary antigen test result. One patient did not receive anti-legionella treatment at all because the diagnosis was determined after death.

In 12 patients, empirical treatment against pathogens other than legionella was stopped within 24 hours of establishing the aetiology of the disease. In four additional patients, this inappropriate treatment was stopped before the availability of the urinary antigen test result. In 10 patients, non-legionella treatment was continued despite the positive urinary antigen test. One patient died before the diagnosis was achieved.

In the control group (54 patients), 16 patients received initial antibiotic treatment with legionella active (two with fluoroquinolones, 14 with macrolides associated either with betalactams or cephalosporins). However, the duration of the combination treatment with macrolides was less than 10 days, as was treatment with the fluoroquinolones, because of the negative result of the urinary antigen test. Five patients did not receive antibiotic treatment. Thirty three patients received antibiotics that did not cover legionella and no antibiotics with legionella coverage were added.

\section{Follow up}

The median length of hospitalisation was 23 days (range, 5-74). Fifteen patients were hospitalised at the intensive care unit because they required ventilator support. Three patients in the intensive care unit died from legionella pneumoniatwo had an antibiotic coverage for legionella and one did not. For this last patient, the positive urinary antigen was available only after death. No relapse occurred. In survivors, the median duration of treatment with legionella active antibiotics was 21 days (range, 15-32).

\section{DISCUSSION}

This retrospective evaluation showed that the legionella urinary antigen test had a major impact on everyday patient management. Most importantly, a positive urinary antigen test prompted the withdrawal of antibiotic treatment directed at non-legionella pathogens. This is a crucial issue, because restricting antibiotic treatment helps to reduce potential adverse effects, the development of antibiotic resistance, and treatment costs.

In the control group, 16 of the 54 patients received antibiotics with legionella coverage, but the treatment duration $(<10$ days) was not adapted for the treatment of legionella pneumonia because of the negative urinary antigen result. For the other control group patients, no legionella specific antibiotic coverage was administered.

\section{"Most importantly, a positive urinary antigen test prompted the withdrawal of antibiotic treatment directed at non-legionella pathogens"}

In addition, the results of the legionella urinary antigen test prompted the initiation of an effective treatment against legionellosis, although this was of less importance because most of our patients with legionnaires' disease had already received antibiotics active against legionella empirically at the time the test result became available. This is in accordance with international guidelines advising that legionella specific antibiotics should be given to patients with community
Take home messages

- In over two thirds of cases, the urinary legionella antigen test had a direct impact on the clinical management of pulmonary legionellosis

- In seven patients, the test results prompted a legionella specific treatment, whereas in 12 cases, non-specific antibiotics were stopped within 24 hours

- Thus, the urinary antigen can have a direct impact on clinical management of pulmonary legionellosis, although patient comorbidities and individual clinical judgment are still important for determining the best treatment to be given in each individual case

acquired pneumonia if some relevant clinical or epidemiological findings suggest this aetiology. ${ }^{5}$ For nosocomial pneumonia, however, the first antibiotic choice does not necessarily include a legionella specific treatment. ${ }^{5}$ In the four nosocomial cases documented in our study, such coverage was provided even though nosocomial legionellosis occurred infrequently at the study hospital.

In the control group, 16 of the 54 the patients received initial antibiotic treatment with legionella coverage, but the duration of the treatment with macrolides, alone or in combination, or with fluoroquinolones, was less than 10 days because of the negative result of the urinary antigen test. Treatment duration is another reason why establishing a definitive diagnosis is of importance. Legionnaires' disease requires a longer antibiotic course than most of the more common causes of community acquired pneumonia. ${ }^{5}$ Failure to use a longer course could result in relapse.

As with any case series, our study findings are restricted to diagnosed cases. The low proportion of positive test results among all tests reveals the propensity of clinicians to rule out legionella as the cause of pneumonia. Furthermore, it is understood that urinary antigen tests have several shortcomings that are not evident in the results reported here. Such tests have reduced sensitivity in the first days after infection and in detecting non-L pneumophila serogroup $\mathrm{l}$ strains, and they lack the power to elucidate the strain related source of infection. For this reason, even though the results of our study support the use of the urinary antigen test, classic culture techniques for use in diagnosis are not to be neglected.

In conclusion, in over two thirds of cases, the urinary antigen had a direct impact on the clinical management of pulmonary legionellosis. However, other patient comorbidities and individual clinical judgment will continue to be important for determining optimal treatment for each individual case.

\section{Authors' affiliations}

J Garbino, I Uçkay, S Fonseca, H Sax, Division of Infectious Diseases, Department of Internal Medicine, University of Geneva Hospitals, 1211 Geneva 14, Switzerland

J-E Bornand, Clinical Virology Laboratory, Department of Internal Medicine, University of Geneva Hospitals

\section{REFERENCES}

1 Stout JE, Yu VL. Legionellosis. N Engl J Med 1997;337:682-7.

2 Tkatch LS, Kusne S, Irish WD, et al. Epidemiology of legionella pneumonia and factors associated with legionella-related mortality at a tertiary care center. Clin Infect Dis 1998;27:1479-86.

3 Tan MJ, Tan JS, Hamor RH, et al. The radiologic manifestations of legionnaire's disease. The Ohio community-based pneumonia incidence study group. Chest 2000;117:398-403. 
4 Waterer GW, Baselski VS, Wunderink RG. Legionella and communityacquired pneumonia: a review of current diagnostic tests from a clinician's viewpoint. Am J Med 2001;110:41-8.

5 Mandell LA, Bartlett JG, Dowell SF, et al. Update of practice guidelines for the management of community-acquired pneumonia in immunocompetent adults. Clin Infect Dis 2003;37:1405-33.

6 Harrison T, Uldum S, Alexiou-Daniel S, et al. A multicenter evaluation of the Biotest legionella urinary antigen EIA. Clin Microbiol Infect 1998;4:359-65.
7 Helbig JH, Uldum S, Bernander S, et al. Clinical utility of urinary antigen detection for diagnosis of community-acquired, travel-associated and nosocomial legionnaires' disease. J Clin Microbiol 2003;41:838-40.

8 Bartlett JG, Breiman RF, Mandell LA, et al. Community-acquired pneumonia in adults: guidelines for management. Clin Infect Dis 1998;26:811-38.

9 Centers for Disease Control and Prevention. Guideline for prevention of nosocomial pneumonia. Respir Care 1994;39:1191-236.

10 Sax H, Dharan S, Pittet D. Legionnaires' disease in a renal transplant recipient: nosocomial or home-grown? Transplantation 2002;74:890-2.

\section{Clinical Evidence-Call for contributors}

Clinical Evidence is a regularly updated evidence-based journal available worldwide both as a paper version and on the internet. Clinical Evidence needs to recruit a number of new contributors. Contributors are healthcare professionals or epidemiologists with experience in evidence-based medicine and the ability to write in a concise and structured way.

Areas for which we are currently seeking authors:

- Child health: nocturnal enuresis

- Eye disorders: bacterial conjunctivitis

- Male health: prostate cancer (metastatic)

- Women's health: pre-menstrual syndrome; pyelonephritis in non-pregnant women

However, we are always looking for others, so do not let this list discourage you.

Being a contributor involves:

- Selecting from a validated, screened search (performed by in-house Information Specialists) epidemiologically sound studies for inclusion.

- Documenting your decisions about which studies to include on an inclusion and exclusion form, which we keep on file.

- Writing the text to a highly structured template (about 1500-3000 words), using evidence from the final studies chosen, within 8-10 weeks of receiving the literature search.

- Working with Clinical Evidence editors to ensure that the final text meets epidemiological and style standards.

- Updating the text every six months using any new, sound evidence that becomes available. The Clinical Evidence in-house team will conduct the searches for contributors; your task is simply to filter out high quality studies and incorporate them in the existing text.

- To expand the topic to include a new question about once every 12-18 months.

If you would like to become a contributor for Clinical Evidence or require more information about what this involves please send your contact details and a copy of your CV, clearly stating the clinical area you are interested in, to Klara Brunnhuber (kbrunnhuber@ bmigroup.com).

\section{Call for peer reviewers}

Clinical Evidence also needs to recruit a number of new peer reviewers specifically with an interest in the clinical areas stated above, and also others related to general practice. Peer reviewers are healthcare professionals or epidemiologists with experience in evidence-based medicine. As a peer reviewer you would be asked for your views on the clinical relevance, validity, and accessibility of specific topics within the journal, and their usefulness to the intended audience (international generalists and healthcare professionals, possibly with limited statistical knowledge). Topics are usually 1500-3000 words in length and we would ask you to review between 2-5 topics per year. The peer review process takes place throughout the year, and our turnaround time for each review is ideally 10-14 days.

If you are interested in becoming a peer reviewer for Clinical Evidence, please complete the peer review questionnaire at www.clinicalevidence.com or contact Klara Brunnhuber (kbrunnhuber@bmigroup.com). 